\title{
Modelling and calibration for cyclic soil-structure interface behaviour
}

\author{
Borana Kullolli1 ${ }^{1,}$, Pablo Cuéllar ${ }^{1}$, Matthias Baeßler ${ }^{1}$ and Hans Henning Stutz ${ }^{2}$ \\ ${ }^{1}$ Bundesanstalt für Materialforschung und -prüfung (BAM), Berlin, Deutschland \\ ${ }^{2}$ Aarhus University, Department of Engineering, Aarhus, Denmark
}

\begin{abstract}
The structural performance of many geotechnical systems (e.g. axially-loaded pile foundations), depends on the shearing resistance at the soil interface, which may govern the load bearing capacity of the foundation. Experimental investigations have shown that this interaction is mainly localised within a narrow shear band next to the structure. Under cyclic loading, a contraction of the soil at the interface may arise (net volume loss), possibly leading to a stress relaxation and thus to a reduction of the load bearing capacity (the so-called friction fatigue). Based on the constitutive similarities between soil continua and interfaces, we propose here the adaption of a Generalized Plasticity model for sandy soils for the numerical analysis of interface problems. In this contribution, the results of an experimental campaign for the parameter calibration of the constitutive model are presented. The tests have been conducted with a ring shear device involving different normal stresses, roughness of the steel plates as well as cyclic loading. The new modelling approach shows promising results and has the additional practical advantage that the interface zone and the soil continuum can both be described with the same constitutive model in general boundary value problems.
\end{abstract}

\section{Introduction}

Interface behaviour involves complex phenomena, which are related to different factors such as roughness of the contact body [1], normal stress at the interface [2, 3], soil density [4] and soil grading [5]. Previous research [1, 4, 6] observed that the thickness of the shear zone at soilstructure interfaces is generally between 5 to 10 times the mean particle size $\mathrm{D}_{50}$.

Among the above mentioned influences, the roughness of the foundation has a major influence for the interface shear behaviour [7]. Experimental observations for dense sand against rough surfaces have typically shown general behaviours like contraction, dilatancy, hardening, and softening [3] under. The shear behaviour of rough interfaces is further characterised by an asymptotic state at large shearing displacement. For smooth interfaces in a dense sand state it can be observed that the shear stress increases sharply at the beginning of the shear test, then it decreases slightly before reaching the residual state, while for loose sands the behaviour is overall contractive [8]. It is important to note that with rough structural interfaces, the shear failure takes place in the soil while for smooth surfaces failure occurs close to the structural interface.

Numerically, the interface has often been modelled through special elements such as interface, contact or joint elements. The interface and joint elements can be broadly divided in two groups: the zero-thickness elements $[9,10]$ and the thin layer approach $[11,12]$. Both kinds of elements can be used to simulate the interface shear behavior in finite-element simulations. Recent advances in interface elements include for instance the hydromechanical coupling effect [13] or the gap opening in cohesive soils [14].

Concerning the specific interface constitutive features such as phase transformation (from hardening to softening) and cyclic degradation, they can be modelled adopting appropriate interface material models. In this respect, the commonly used model of a linearly-elasticperfectly-plastic interface with Coulomb's friction cannot reproduce important phenomena concerning both the shear and the volumetric behavior of the interface, which require the use of more advanced models (e.g. [15-18]).

In this paper, we use the methodology proposed by Stutz et al. [19], which advocates the use of 3D constitutive models in a reduced form as interface models. We employ here an adaption of the Pastor-Zienkiewicz model for sands in the frame of the theory of the generalized plasticity [20]. Due to analogy between the behavior of soil continuum and the soil-structure interface [3], it is possible to use the same constitutive law in both zones through the adaptation of the model to simple shear conditions.

To calibrate the interface model, a set of experimental tests has been performed with a ring shear device. The specimen was tested with three different normal stresses, four different roughness surfaces from smooth to rough and a cyclic test under constant normal load conditions.

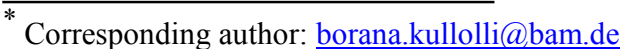




\section{Interface constitutive model}

\subsection{Generalized Plasticity framework for sands}

The Generalized-Plasticity was developed by Zienkiewcz \& Mroz [21] and extended and improved to reproduce sand behaviour by Pastor et al. [22-24]. One advantage of this model is that no plastic potential or yield surfaces needs explicitly to be defined, as the material behaviour is described in terms of two direction vectors, namely the loading vector $n$ and the plastic flow direction vector $n_{g}$.

The material behaviour is defined in its basic form by the stress-strain relationship, given as:

$$
\begin{gathered}
d \sigma=D^{e p} \cdot d \varepsilon \\
d \sigma=\left(D^{e}-\frac{D^{e}: n_{g L / U}: n^{T}: D^{e}}{H_{L / U}+n^{T}: D^{e} n_{g L / U}}\right) \cdot d \varepsilon
\end{gathered}
$$

where $D^{e p}$ is the elasto-plastic stiffness matrix, $D^{e}$ is the elastic stiffness matrix and $H$ is the plastic modulus. The subscripts $L$ and $U$ discriminate between loading and unloading conditions as:

$$
\begin{array}{ll}
\boldsymbol{d} \boldsymbol{\sigma}^{\boldsymbol{T}} \cdot \boldsymbol{n}>0 & \text { Loading } \\
\boldsymbol{d} \boldsymbol{\sigma}^{\boldsymbol{T}} \cdot \boldsymbol{n}<0 & \text { Unloading } \\
\boldsymbol{d} \boldsymbol{\sigma}^{\boldsymbol{T}} \cdot \boldsymbol{n}=0 & \text { Neutral }
\end{array}
$$

The plastic flow direction $n_{g}$ can be decomposed in its volumetric $n_{g, v}$ and shear component $n_{g, s}$ :

$$
\begin{gathered}
\boldsymbol{n}_{\boldsymbol{g}}=\left\{\begin{array}{l}
n_{g, v} \\
n_{g, s}
\end{array}\right\} \\
n_{g, v}=\frac{d_{g}}{\sqrt{1+d_{g}^{2}}} \\
n_{g, s}=\frac{1}{\sqrt{1+d_{g}^{2}}}
\end{gathered}
$$

Based on the experimental results obtained by [23, 25] the proposed form for the dilatancy $d_{g}$ is:

$$
d_{g}=\left(1+\alpha_{g}\right) \cdot\left(M_{g}-\eta\right)
$$

The dilatancy $d_{g}$ depends on the stress ratio $\eta=q / p$ between deviatoric $q$ and confining stresses $p$ and the slope $M_{g}$ of the critical state line in the $p-q$ plane. The parameter $\alpha_{g}$ is a material constant obtained by experimental diagrams of dilatancy $d_{g}$ versus stress ratio.

Similar to $n_{g}$, the load vector $n$ is defined by $M_{f}$ and $\alpha_{f}$ which are constitutive parameters. The model does not have a notion for the yield surface, but the failure surface is defined implicitly by:

$$
\begin{array}{r}
\boldsymbol{n}=\left\{\begin{array}{l}
n_{v} \\
n_{s}
\end{array}\right\} \\
n_{v}=\frac{d_{f}}{\sqrt{1+d_{f}^{2}}}
\end{array}
$$

$$
n_{s}=\frac{1}{\sqrt{1+d_{f}^{2}}}
$$

where:

$$
d_{f}=\left(1+\alpha_{f}\right) \cdot\left(M_{f}-\eta\right)
$$

The elastic matrix is assumed uncoupled with respect to its volumetric and shear components, i.e. the elastic strains are here assumed to be non-dilatant.

$$
\boldsymbol{D}^{e}=\left[\begin{array}{cc}
K^{e} & 0 \\
0 & G^{e}
\end{array}\right]
$$

The elastic shear modulus $G^{e}$ and the bulk modulus $K^{e}$ both depend on the confining stress $p$, normalized here by a reference stress $p_{0}$; whereby $K_{0}{ }^{e}$ and $\mathrm{G}_{0}{ }^{e}$ are the bulk and shear moduli at the reference stress state.

$$
\begin{aligned}
K^{e} & =K_{0}^{e} \frac{p}{p_{0}} \\
G^{e} & =G_{0}^{e} \frac{p}{p_{0}}
\end{aligned}
$$

The plastic modulus $H_{L}$ during loading, permits the consideration of different aspects of sand behaviour like the existence of a critical state line where the residual stress states are present.

$$
H_{L}=H_{0} \cdot p \cdot H_{f} \cdot\left(H_{v}+H_{s}\right) \cdot H_{D M}
$$

$H_{L}$ incorporates the following ingredients: (i) a stress dependence through the effective confining stress $p$, (ii) an isotropic plastic modulus $H_{0}$, (iii) a frictional factor $H_{f}$ that limits the possible stress states within the sand, (iv) a volumetric strain hardening function $H_{v},(\mathrm{v})$ a deviatoric strain hardening $H_{s}$, which models the material degradation by accumulated strains, and finally (vi) $H_{D M}$ a discrete memory factor that accounts for previous loading.

For the unloading case the plastic modulus can be defined by a simple expression depending on the point where load reversal takes place as proposed by [24]

$$
H_{U}=H_{U, 0} \cdot\left(\frac{M_{g}}{\eta_{u}}\right)^{\gamma_{U}}
$$

where $\eta_{U}$ is the stress ratio at the beginning of the unloading phase and $\gamma_{U}$ is plastic unloading parameter.

The described model was implemented in a finite element code (GeHoMadrid) where simulations for coupled hydro-mechanical problems in 2D [26, 27] and $3 \mathrm{D}$ [28] boundary value problems were simulated.

\subsection{Model adaptation for interfaces}

In general, the soil behaviour can be described through the critical state soil mechanics (CSSM) [29], which postulates a critical state of soil at large deformation where shear deformation continues without dilatancy and change of the stress ratio. 
a)
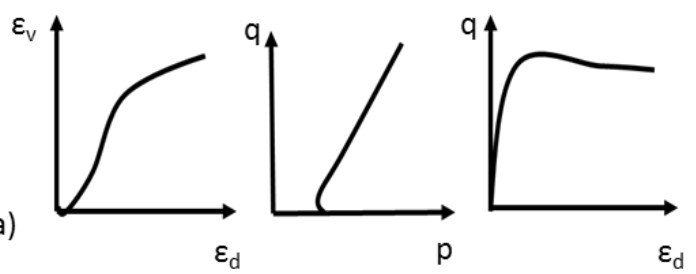

b)
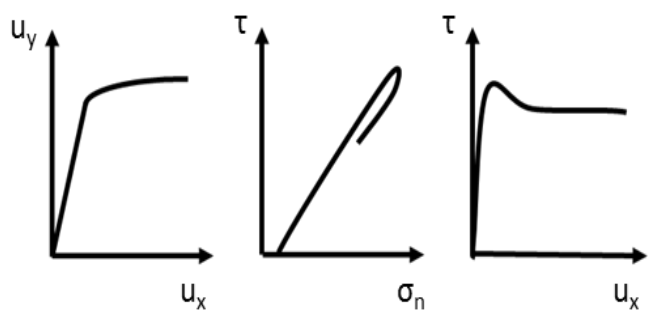

Fig. 1. Similarities between (a) Triaxial Results (b) Interface test (adapted from [3])

Due to the analogies between the behaviour of sand and interface (Figure 1), it appears sensible to use the concept of CSSM to model the soil-structure interface behaviour (e.g. [15-18]). In this respect, certain modifications are needed: the confining stress $p$, the deviatoric stress $q$, the volumetric $\varepsilon_{v}$ and deviatoric strains $\varepsilon_{d}$ of CSSM are replaced by the normal stress of interface test $\sigma_{n}$, the shear stress $\tau$, the shear displacement $u_{x}$ and normal displacements $u_{y}$. As results these changes affect the parameters where they are present such as the stress ratio $\eta$, the bulk modulus $K^{e}$ and shear modulus $G^{e}$ :

$$
\begin{gathered}
\eta=\frac{\sigma_{n}}{\tau} \\
K^{e}=K_{0}^{e} \frac{\sigma_{n}}{\sigma_{n 0}} \\
G^{e}=G_{0}^{e} \frac{\sigma_{n}}{\sigma_{n 0}}
\end{gathered}
$$

\section{Experimental Setup}

The tests are performed with a Bromhead ring shear test device as in Figure 2, which has the advantage of the nonrestricted shear displacements. Moreover, the boundary effect is also reduced compared to the direct shear device as the ring shape avoid stress concentration due to the absence of sharp edges in the shearing direction [1]. The normal load is applied by the step motor installed in the upper cylinder, while the rotational displacements are applied by the motor under the device. Once the sample is mounted into the ring, a vertical cylinder (fixed under the load cell) is applied at the top of it to exert the normal stress. After the consolidation period ( $\mathrm{t}$ 95 $=3$ min with a dry specimen), the shearing test phase is started.

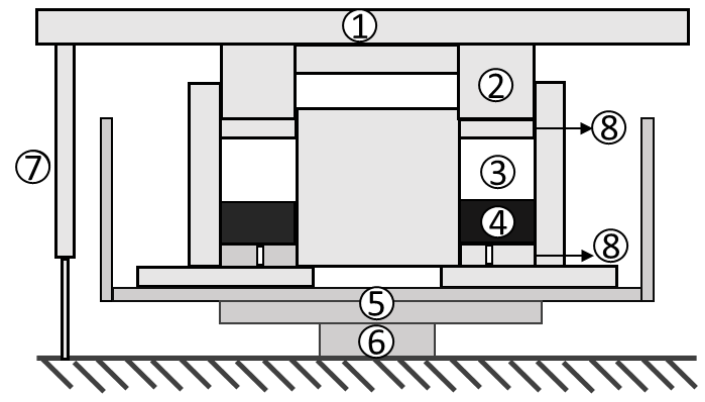

Fig. 2. Ring shear device sketch

1-Load cell 2-Normal Load solid cylinder 3-Sand 4-Steel plate/sand 5-Rotating base 6-Stand 7-Vertical displacement sensor 8-Porous stone

The sand is a sub-rounded quartz sand $\left(97 \% \mathrm{SiO}_{2}\right)$ and mixed in the lab to ensure repeatability of tests of the soil material. The diameter varies from $0.06-4 \mathrm{~mm}$, where $\mathrm{D}_{50}=0.55 \mathrm{~mm}$. The grain size distribution can be found in Figure 3.

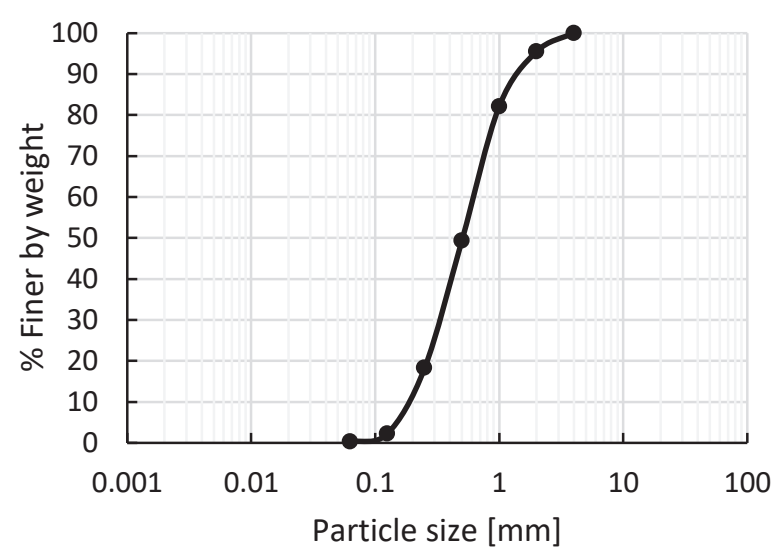

Fig. 3. Grain size distribution of the used sand

The specimen is built in three layers with the same mass. The desired density is achieved through compaction with a heavy load. The target relative density of the sand was chosen as $\mathrm{Dr}=65 \%$.

For the experiments, four steel plates (Figure 4) with different roughness have been used (r1, r2, r3, r4, where $\mathrm{r} 1$ is the smoothest and $\mathrm{r} 4$ is the roughest). The respective values of the maximal roughness $R_{\max }$, which is defined as vertical distance between highest and lowest peak for a characteristic length $\mathrm{L}=2,5 \mathrm{~mm}$, are given in Table 1 . Additional soil-vs-soil ring shear tests have also been performed to compare them with the interface shear results.

Table 1. Roughness values

\begin{tabular}{|l|l|}
\hline Plate Name & $\mathrm{R}_{\max }[\mu \mathrm{m}]$ \\
\hline r1 & 14,34 \\
\hline r2 & 33,31 \\
\hline r3 & 53,48 \\
\hline r4 & 149,41 \\
\hline
\end{tabular}




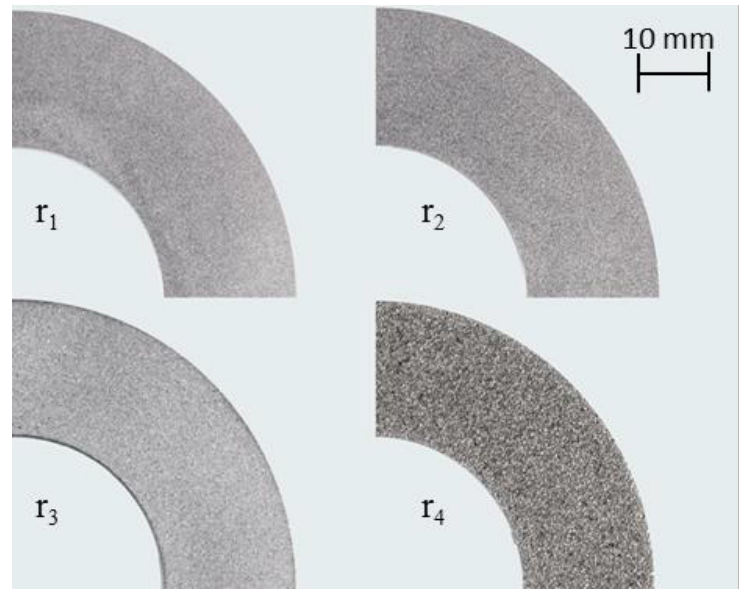

Fig. 4. Steel rings from smoothest to roughest $\left(r_{1}-r_{4}\right)$

\section{Results and discussion}

\subsection{Calibration Process}

The calibration procedure is as recommended [21] starts by fitting the elastic slope through $K_{e}$ and $G_{e} . \beta_{0}$ and $\beta_{1}$ have an important role on defining the peak behaviour while the value of $M_{f}$ defines the shear stress residual plateau. For simplicity, $M_{g}$ is here fixed to be equal to $D r * M_{f}$. The roughness effect and normal stress variation can then be calibrated through modification of $M_{f}$ and $\beta_{0}$ as in table 2 and 3 . . The cyclic parameters $H_{U, 0}$ and $\gamma_{u}$ are defined by curve fitting the numerical results in the shear displacement vs. shear stress experimental results.

Table 2. Parameter calibration for Fig. 5. and Fig. 7.

\begin{tabular}{|c|c|c|c|c|c|}
\hline & $\mathrm{R} 1$ & R2 & R3 & $\mathrm{R} 4$ & SS \\
\hline$K_{e}[k P a]$ & 3000 & 3000 & 3000 & 3000 & 3000 \\
\hline$G_{e}[k P a]$ & 8000 & 8000 & 8000 & 8000 & 8000 \\
\hline$M_{f}$ & 0.41 & 0.51 & 0.59 & 0.65 & 0.72 \\
\hline$\alpha_{f} / \alpha_{g}$ & 0.45 & 0.45 & 0.45 & 0.45 & 0.45 \\
\hline$M_{g}$ & 0.26 & 0.33 & 0.38 & 0.42 & 0.47 \\
\hline$H_{0}[\mathrm{kPa}]$ & 600 & 600 & 600 & 600 & 6000 \\
\hline$\beta_{1}$ & 35.2 & 35.2 & 35.2 & 35.2 & 35.2 \\
\hline$\beta_{0}$ & 0.001 & 0.001 & 0.005 & 0.014 & 0.015 \\
\hline \multicolumn{5}{|l|}{ Cyclic } & \\
\hline$r$ & & & & 2 & \\
\hline$H_{u 0}$ & & & & 50 & \\
\hline$r_{U}$ & & & & 10 & \\
\hline
\end{tabular}

Table 3. Parameter calibration for Fig. 6.

\begin{tabular}{|l|l|l|l|}
\hline $\mathrm{R} 3$ & $\begin{array}{l}\sigma=100 \\
\mathrm{kPa}\end{array}$ & $\begin{array}{l}\sigma=200 \\
\mathrm{kPa}\end{array}$ & $\begin{array}{l}\sigma=300 \\
\mathrm{kPa}\end{array}$ \\
\hline$K_{e}[\mathrm{kPa}]$ & 3000 & 3000 & 3000 \\
\hline$G_{e}[\mathrm{kPa}]$ & 8000 & 8000 & 8000 \\
\hline$M_{f}$ & 0.32 & 0.59 & 1.03 \\
\hline$\alpha_{f} / \alpha_{g}$ & 0.45 & 0.45 & 0.45 \\
\hline$M_{g}$ & 0.21 & 0.38 & 0.67 \\
\hline$H_{0}[\mathrm{kPa}]$ & 600 & 600 & 600 \\
\hline$\beta_{I}$ & 35.2 & 35.2 & 35.2 \\
\hline$\beta_{0}$ & 0.005 & 0.005 & 0.007 \\
\hline
\end{tabular}

\subsection{Roughness effect}

In Figure 5 the effect of the roughness on shear stress and normal displacement evolution is shown.
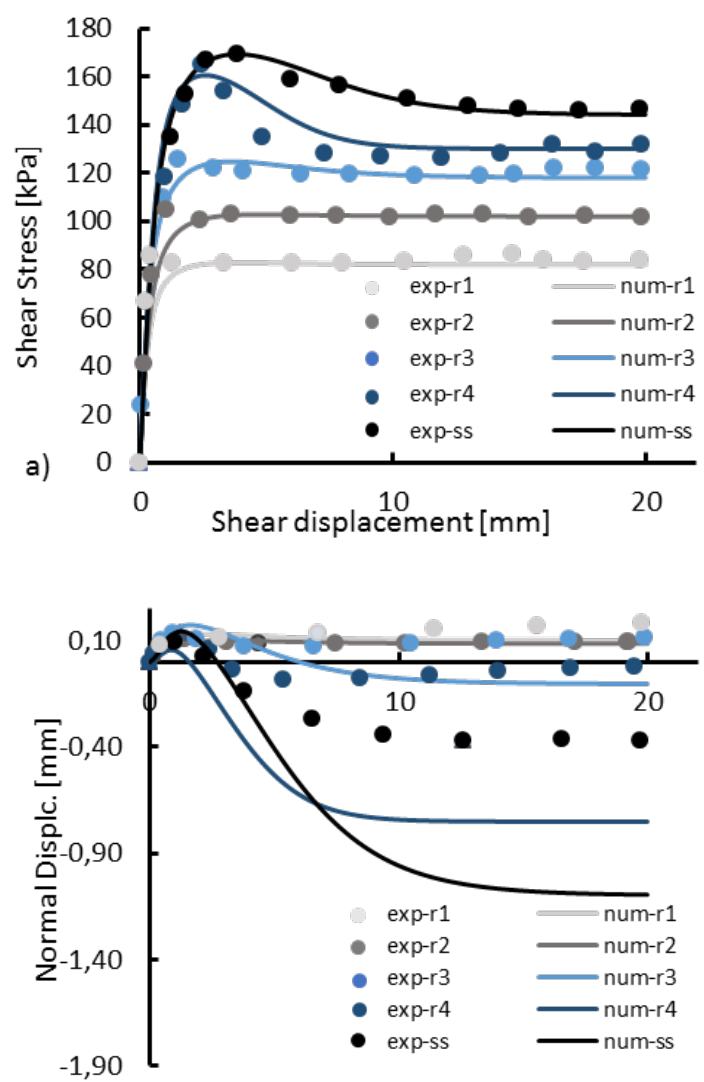

b)

Shear displacement [mm]

Fig. 5. Evolution of shear stress and normal displacement with roughness variation. Experimental results: solid markers, Numerical results: continuous lines. SS: soil-vs-soil shear test. Sign convention for normal displacements: positive -downward (settlement), negative - heave

The applied normal stress is $\sigma_{n}=200 \mathrm{kPa}$, while the maximum shear displacement is set to $20 \mathrm{~mm}$. The peak shear stress evolves with increase of roughness. For the smoothest case, after reaching the peak the shear stress value remains rather constant and the settlements show a purely contractive behaviour. The increase of roughness from $r_{3}$ to soil-soil shearing leads in the development of a peak in the shear-stress deformation response. This can be explained by a more pronounced dilation and a larger soil volume that becomes mobilized in the shear process. The discrepancy between numerical and experimental results could be overcomed if $\mathrm{M}_{\mathrm{g}}$ is not chosen according to the relation $D_{r} * M_{f}$. However, having this relation has eased the calibration process by considering only two parameters for calibration.

\subsection{Normal stress effect}

Tests with different normal stress levels (100, 200, 400 $\mathrm{kPa}$ ) have been performed with the rough interface ring $\left(\mathrm{r}_{3}\right)$. With an increase in normal stress, the shear stress increases accordingly (Figure 6a). The volumetric response (Figure $6 \mathrm{~b}$ ) shows an initial contractive followed by dilative behaviour which is less dominant when normal 
stress increase (in accordance with CSSM). However numerically the response of the model showed a larger discrepancy with the experimental results for the same reason mentioned in section 4.3 , while the shear stress response is satisfactorily reproduced.
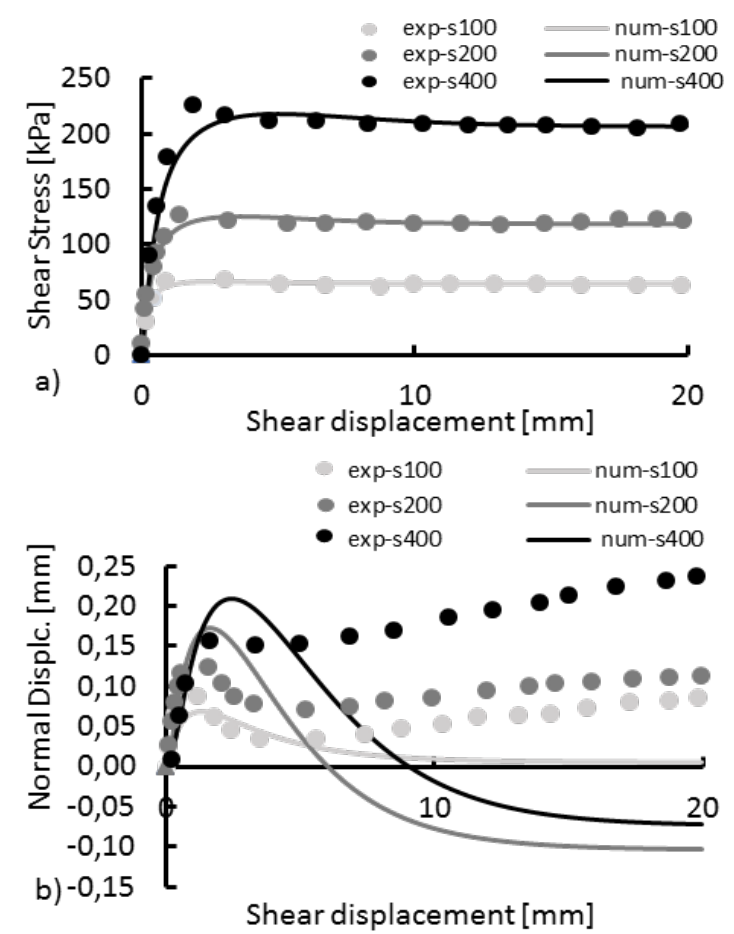

Fig. 6. Evolution of shear stress and normal displacement with normal stress variation. Experimental results in solid markers, Numerical results in continuous lines. Sign convention for normal displacements: positive -downward (settlement), negative - heave.

\subsection{Cyclic Loading}

Beside the monotonic response of the model it is also important to consider the cyclic response. Figure 7 shows the test results with the roughest steel plate $\left(r_{4}\right)$ under
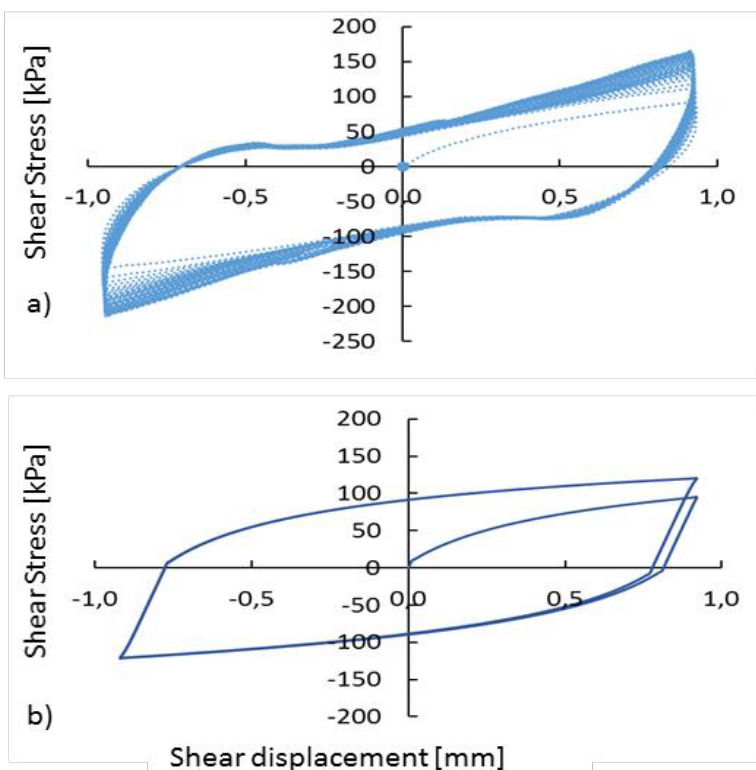

constant normal load conditions (CNL) for 50 cycles. The cyclic test has a shearing amplitude of $\Delta \mathrm{u}= \pm 1 \mathrm{~mm}$ and the same normal stress as in section 4.2 The experimental results show a densification with the increase of cycles (Figure 7 a), which is apparent for both graphs with the shear stress (progressive hardening) and normal displacements (progressive settlement). The numerical model (Figure $7 \mathrm{~b}$ ) captures well the initial behaviour and cyclic reversal behaviour. However, the interface model still lacks the ability to simulate the effects of densification in an appropriate way without recalibrating the parameters from the monotonic tests (since no state parameters are included in the model yet).

\section{Conclusions}

In the present study, a material model for sand has been adapted as an interface model. To calibrate the model, an experimental campaign with a ring shear device has been performed. The numerical results show a good agreement with experimental results considering varying roughness and levels of normal stress. In addition, the model is able to simulate important features of the cyclic interface behaviour. The experimental test results clearly indicate that the interface model can be further adapted by introducing a parameter to simulate different roughness conditions at the interface. Suitable candidates for these modifications are the critical state slope parameter $M_{f}$ and the strain hardening parameter $\beta_{0}$. The adaption of the model would lead to a further improvement of the application potential for the model due to the fact that the parameterisation for different simulations could be simplified.

The presented model is being developed and implemented in the academic numerical FE code GeHoMadrid, developed by the authors and coworkers in the technical universities of Madrid and Hohai, and by researchers of CEDEX in Madrid. The collective work carried out by all these researchers makes the
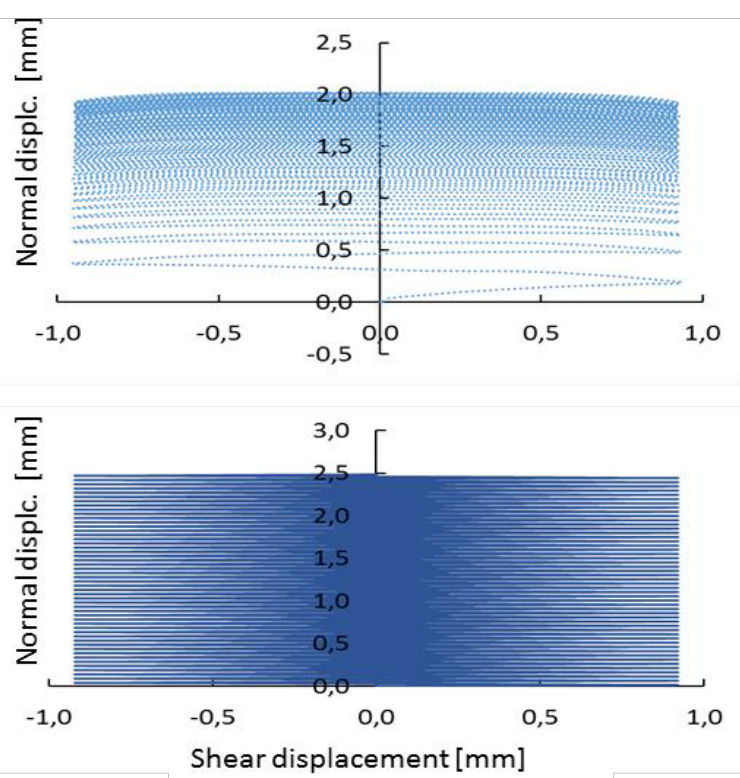

Fig. 7. Evolution of shear stress and normal displacement for cyclic loading a) Experimental results-points, b) Numerical results continuous line. Sign convention for normal displacements: positive -downward (settlement) 
present investigations possible and is here gratefully acknowledged. The authors would also like to thank the helpful discussions with Manuel Pastor, and Miguel Martin Stickle (Universidad Politecnica de Madrid), Pablo Mira (CEDEX in Madrid) and Franck Rackwitz, Ralf Glasenapp and Viet Hung Le from the Technical University of Berlin.

\section{References}

1. H. Kishida, M. Uesugi, Geotechnique, Tests of the interface between sand and steel in the simple shear apparatus, 37(1), 45-52, (1987).

2. M. Boulon, Comp. and Geotechnique. Basic features of soil structure interface behaviour, 7(1-2), 115-131, (1989).

3. M. Boulon, R. Nova, Comp. and Geotechnics. Modelling of soil-structure interface behaviour a comparison between elastoplastic and rate type laws, 9(1-2), 21-46, (1990).

4. J. Dejong, D. White, M. Randolph, Soils and Foundations, Microscale observation and modeling of soil-structure interface behavior using particle image velocimetry, 46(1), 15-28, (2006).

5. M. Uesugi, H. Kishida, Y. Tsubakihara, Soils and foundations, Behavior of sand particles in sand-steel friction, 28(1), 107-118, (1988).

6. S. Georgi, Experimentelle Untersuchungen $\mathrm{zu}$ Verformungsakkumulation und Tragfähigkeitsreduktion zyklisch belasteter Pfalgründungen', Phd Thesis. Technical University of Berlin, (2016).

7. V. Fioravante, Soils and foundations, On the shaft friction modelling of non-displacement piles in sand, 42(2), 23-33, (2002).

8. I. Shahrour, F. Rezaie, Comp. and Geotechnics. An elastoplastic constitutive relation for the soilstructure interface under cyclic loading, 21(1), 21-39, (1997).

9. R.E. Goodman, R.L. Taylor, T.L. Brekke, J. of Soil Mech.s \& Found. Div, A model for the mechanics of jointed rocks, (1968).

10. G. Beer, Int. J. for num. meth. in eng., An isoparametric joint/interface element for finite element analysis, 21(4), 585-600, (1985).

11. C. Desai, M. Zaman, J. Lightner, H. Siriwardane, Int. J.for Num. and Anal. Meth. in Geomechanics, Thinlayer element for interfaces and joints, 8(1), 19-43, (1984).

12. D. Griffiths, Proc. of the 5th Int. Con. on Num. Meth. in Geomech., Nagoya, Numerical modelling of interfaces using conventional finite elements, 2837 844, (1985).

13. B.Cerfontaine, A.-C.Dieudonné, J.-P. Radu, F. Collin, R.Charlier, Comp. and Geotech., 3d zerothickness coupled interface finite element:69 124140, (2015).
14. H. Stutz, F. Wuttke, T. Benz, Num. Meth. in Geotech. Eng., Extended zero-thickness interface element for accurate soil-pile interaction modelling, 283, (2014).

15. H. Liu, E. Song, H.I. Ling, Mech. Research Comm., Constitutive modeling of soil-structure interface through the concept of critical state soil mechanics, 33(4), 515-531, (2006).

16. J. Liu, D. Zou, X. Kong, Comp. and Geotech., A three-dimensional state-dependent model of soilstructure interface for monotonic and cyclic loadings, 61 166-177, (2014).

17. G. Mortara, Politech. di Torino, An elastoplastic modelling sand structure interface behaviour under monotonic and cyclic loading, (2003).

18. H.H. Stutz, F. Wuttke, Journal of Zhejiang Univ. A, Hypoplasticmodeling of soil-structure interfaces in offshore applications, 19(8), 624-637, (2018).

19. H. Stutz, D. Mašín, F. Wuttke, Acta Geotech., Enhancement of a hypoplastic model for granular soil-structure interface behaviour, 11(6), 1249-1261, (2016).

20. B. Kullolli, H.H. Stutz, P. Cuellar, M. Baessler, F. Rackwitz, Numge , A generalized plasticity model adapted for shearing interface problems, 97, (2018).

21. O. Zienkiewicz, Z. Mroz, Mech. of Eng. materials, Generalized plasticity formulation and applications to geomechanics, 44(3), 655-680, (1984).

22. M. Pastor, O. Zienkiewicz, K. Leung, Int. J. for Num. and Analyt. Meth. in Geomech., Simple model for transient soil loading in earthquake analysis. Ii. Nonassociative models for sands, 9(5), 477-498, (1985).

23. M. Pastor, O. Zienkiewicz, Proc., 2nd Int. Symp. on Num. Models in Geomech., A generalized plasticity, hierarchical model for sand under monotonic and cyclic loading, 131-150, (1986).

24. M. Pastor, O. Zienkiewicz, A. Chan, Int. Journal for Num. and Analyt. Methods in Geomechanics, Generalized plasticity and the modelling of soil behaviour, 14(3), 151-190, (1990).

25. E. Frossard, Géotechnique, Une équation d'éoulement simple pour les matériaux granulaires, 33(1), 21-29, (1983).

26. P. Mira, M. Pastor, T. Li, X. Liu, Comp. meth. in applied mech. and eng., A new stabilized enhanced strain element with equal order of interpolation for soil consolidation problems, 192(37), 4257-4277, (2003).

27. P. Mira, M. Pastor, T. Li, X. Liu, Revue française de génie civil, Failure problems in soils: An enhanced strain coupled formulation with application to localization problems, 8(5-6), 735-759, (2004).

28. P. Cuellar, Pile foundations for offshore wind turbines: Numerical and experimental investigations on the behavior under short term and long term cyclic loading, PhD Thesis, Tech. Univ. of Berlin, (2011).

29. D.M. Wood, Cambridge University Press, Soil behaviour and critical state soil mechanics, (1990). 